\title{
Review of: "The antagonistic mechanism of Bacillus velezensis ZW10 against rice blast disease: Evaluation of ZW10 as a potential biopesticide"
}

\author{
Zongwang $\mathrm{Ma}^{1}$ \\ 1 Northwest Normal University Lanzhou
}

Potential competing interests: The author(s) declared that no potential competing interests exist.

This paper describes the biological control mechanisms of the strain Bacillus velezensis ZW10 against rice blast pathogen Magnaporthe oryzae, and rice field trials have confirmed that the strain B. velezensis ZW10 is a good biological control agent to $M$. oryzae. The mechanisms involved in the interaction of $B$. velezensis ZW10 and $M$. oryzae are direct antifungal activity against $M$. oryzae, inhibit the appressoria formation of $M$. oryzae, increased production ROS in rice leaves during M. oryzae infection as well as the over expression of the defense related genes and enzymes in rice.

Although the authors have studied diverse mechanisms triggered by a semipurified metabolites in this type of interaction, the chemical components of the semipurified metabolites should be analyzed further, to give clues of the detailed information of the chemical structure of the secondary metabolites produced by $B$. velezensis ZW10 involved in the biological control of $M$. oryzae in rice.

Anyway, this paper could give readers some useful results and research methodology about the biocontrol of M. oryzae. 